\title{
Extracellular vesicles released by the human gut symbiont Bacteroides thetaiotaomicron in the mouse intestine are enriched in a selected range of proteins that influence host cell physiology and metabolism
}

Regis Stentz ( $\square$ regis.stentz@quadram.ac.uk)

Quadram Institute Bioscience https://orcid.org/0000-0003-1021-9439

Udo Wegmann

University East Anglia

Maria Guirro

Universitat Rovira i Virgili-EURECAT

Will Bryant

University College London

Avani Ranjit

University East Anglia

Andrew J. Goldson

Quadram Institute Bioscience

Arlaine Brion

Quadram Institute Bioscience

Kathryn Cross

Quadram Institute Bioscience

Catherine Booth

Quadram Institute Bioscience

Ariadna Miquel-Clopes

Quadram Institute Bioscience

Emily Jones

Quadram Institute Bioscience

Patrick Gunning

Quadram Institute Bioscience

Padhmanand Sudhakar

Earlham Institute

Dezsó Módos

Earlham Institute 


\section{lan R. Brown}

University of Kent

Tamás Korcsmáros

Earlham Institute

Simon R. Carding

Quadram Institute Bioscience

\section{Research Article}

Keywords: Bacterial extracellular vesicles, proteome, intestine, microbiota, nutrition, Bacteroides thetaiotaomicron

Posted Date: December 10th, 2020

DOl: https://doi.org/10.21203/rs.3.rs-124947/v1

License: (c) (1) This work is licensed under a Creative Commons Attribution 4.0 International License. Read Full License 
1 Extracellular vesicles released by the human gut symbiont Bacteroides thetaiotaomicron in the mouse

2 intestine are enriched in a selected range of proteins that influence host cell physiology and metabolism 3

4 Régis Stentz $^{1^{*}}$, Udo Wegmann ${ }^{2}$, Maria Guirro ${ }^{3}$, Will Bryant ${ }^{4}$, Avani Ranjit ${ }^{1}$, Andrew J. Goldson ${ }^{5}$, 5 Arlaine Brion ${ }^{5}$, Kathryn Cross ${ }^{5}$, Catherine Booth ${ }^{5}$, Ariadna Miquel-Clopes ${ }^{1}$, Emily Jones ${ }^{1}$, Patrick

6 Gunning ${ }^{5}$, Padhmanand Sudhakar ${ }^{6,7}$, Dezső Módos ${ }^{1,6}$, Ian R. Brown ${ }^{8}$, Tamás Korcsmáros ${ }^{1,6}$, Simon 7 R. Carding ${ }^{1,9}$

8

$9 \quad{ }^{1}$ Gut Microbes and Health Research Programme Quadram Institute Bioscience, Norwich, UK

${ }^{2}$ School of Chemistry, University East Anglia, Norwich, UK

${ }^{3}$ Eurecat, Centre Tecnològic de Catalunya, Centre for Omic Sciences (COS), Joint Unit Universitat

Rovira i Virgili-EURECAT, Unique Scientific and Technical Infrastructures (ICTS), Reus, Spain

${ }^{4}$ Great Ormond Street Hospital, University College London, London, UK

$14{ }^{5}$ Core Science Resources Quadram Institute Bioscience, Norwich, UK

${ }^{6}$ Earlham Institute, Norwich, UK

${ }^{7}$ Department of Chronic Diseases, Metabolism and Ageing, TARGID, KU Leuven, Leuven, Belgium

${ }^{8}$ School of Biosciences, University of Kent, Canterbury, UK

${ }^{9}$ Norwich Medical School, University East Anglia, Norwich, UK

*Correspondence :

Régis Stentz.regis.stentz@quadram.ac.uk

Keywords: Bacterial extracellular vesicles, proteome, intestine, microbiota, nutrition, Bacteroides thetaiotaomicron 


\section{Abstract}

It is becoming increasingly clear that bacterial extracellular vesicles (BEVs) produced by members of the intestinal microbiota can contribute to microbe-host cell interactions that impact on host health. A major unresolved question is the nature of the cargo packaged into these BEVs and how they can impact on host cell function. Here we have analysed the proteome of BEVs produced by the major human gut symbiont Bacteroides thetaiotaomicron in both in vitro cultures using defined and complex medias, and in vivo in fed or fasted animals to determine the impact of nutrient stress on the BEV proteome, and to identify proteins specifically enriched in BEVs produced in vivo. In contrast to BEVs produced in vitro where limiting nutrient provision resulted in an increase in a large fraction of proteins, the protein content of BEVs extracted from fasted versus fed mice was less affected with similar numbers of proteins showing increased and decreased abundance. We identified 102 proteins exclusively enriched in BEVs in vivo of which the majority (66/102) were enriched independently of their expression in the parent cells implicating the existence of an active mechanism to drive the selection of a group of proteins for their secretion into BEVs within the intestine. Amongst these abundantly expressed proteins in BEVs in vivo were a bile salt hydrolase and a dipeptidyl peptidase IV that were characterised further and shown to be active and able to degrade host-derived substrates with defined roles in metabolism. Collectively these findings provide additional evidence for the role of BEVs in microbiota-host interactions with their contents playing key roles in the maintenance of intestinal homeostasis, and host metabolism.

\section{Introduction}

The human gastrointestinal (GI) tract accommodates a microbial community (the microbiota) comprising trillions of cells that carry out vital functions for human health. Increasing our understanding of the basis of this mutualistic relationship and its impact on human health and disease is dependent on defining the pathways and mediators of microbiota-host crosstalk. Many studies have identified the importance of microbe- and host-derived soluble factors in this crosstalk [reviewed in 1,2]. More recently, another pathway of host-microbe crosstalk has been identified that involves bacterial extracellular vesicles (BEVs) [3], which contain various macromolecules with the potential of contributing to interactions with other members of the microbial community but also with host cells [4-7].

BEVs represent a novel secretion system enabling the dissemination of membrane-encapsulated cellular materials including proteins, nucleic acids and metabolites into the extracellular milieu $[8,9]$ and beyond [7]. These include membrane vesicles (MVs) produced by Gram-positive bacteria, and outer membrane vesicles (OMVs) and outer-inner membrane vesicles [10-12] produced by Gram-negative bacteria. BEVs produced by pathogenic bacteria have historically been the most intensively investigated. The animal GI 
tract contains a multitude of bacterial species capable of producing membrane vesicles that are implicated in digestion and in the development and functioning of the immune system [5,13,14]. Bacteroides thetaiotaomicron $(\mathrm{Bt})$ is a prominent Gram-negative anaerobe residing in the caecum and colon of most or all animals. The BEVs it produces are small, spherically bilayered $(50-400 \mathrm{~nm})$ vesicles derived from the cell envelope that contain mainly periplasmic contents in their lumen. Proteomic studies have shown that members of the Bacteroides genus, including Bt, use their BEVs as delivery vehicles for the distribution of hydrolases, such as proteases and glycosidases [15] within the lumen of the GI tract [16]. In particular, Bt BEVs can digest polysaccharides [13], phytate and inositol polyphosphate derivatives [16], and modulate the immune system $[5,6,17-20]$. They can access and transmigrate boundary epithelial cells using different routes enabling them to interact with mucosal immune cells and to disseminate more widely via the bloodstream $[7,14,20]$. Our further understanding of BEV biology in general and of their interaction with the host in particular, is dependent on defining the factors that regulate their generation and the cargo they carry $[12,21]$.

We have performed a comparative proteomic analysis of BEVs produced under different nutrient conditions both in vitro and in vivo in the mouse caecum to assess the impact of nutrient availability on BEV protein composition. Differential proteome analysis enabled comparisons of the abundance of each protein identified in BEVs with that in the parent cells. As a result, we identified proteins in BEVs that are determinants in BEV-host interactions and able to play key roles in the maintenance of the intestinal homeostasis and host metabolism.

\section{Materials and Methods}

\section{BEV preparation}

BEVs were isolated following a method adapted from Stentz et al. [22]. The bacterium Bt VPI-5482 was grown anaerobically at $37^{\circ} \mathrm{C}$ with agitation using a magnetic stirrer in either Brain Heart Infusion (BHI) medium (Oxoid/Thermo Fisher, Basingstoke, United Kingdom) or the defined medium, BDM [9], both supplemented with $0.001 \%$ haemin. BHI (three independent cultures) and BDM (three independent cultures) were inoculated with an overnight culture of $\mathrm{Bt}$ at an initial $\mathrm{OD}_{600}$ of 0.05 . After $5 \mathrm{~h}$ in $\mathrm{BHI}$ and $12 \mathrm{~h}$ in BDM (OD approximately 3.0, early stationary phase), the cells were centrifuged at $5500 \mathrm{~g}$ for 45 $\min$ at $4{ }^{\circ} \mathrm{C}$. The cell pellets were rinsed twice with $50 \mathrm{~mL}$ of ice-cold phosphate buffered saline (PBS), $\mathrm{pH}$ 7.4, snap frozen in liquid nitrogen and stored at $-80^{\circ} \mathrm{C}$ prior to extraction. The supernatants were filtered through polyethersulfone (PES) membranes ( $0.22 \mu \mathrm{m}$ pore-size) (Sartorius) to remove debris and cells. The sterility of the vesicle-containing filtrates was confirmed by plating onto BHI-haemin agar. BEVs in the 
$500 \mathrm{ml}$ filtrates were concentrated by crossflow ultrafiltration (100 kDa MWCO, Vivaflow 200, Sartorius) to $0.5 \mathrm{~mL}$, diluted by addition of $500 \mathrm{~mL}$ of ice-cold phosphate buffered saline (PBS), $\mathrm{pH} 7.4$, and the suspensions were concentrated again by crossflow filtration to $0.5 \mathrm{~mL}$ and filter-sterilised through a 0.22 $\mu \mathrm{m}$ PES membrane (Sartorius). Vesicle concentration was determined by Nanoparticle Tracking Analysis (NTA). The volume of the retentate was adjusted to $8.9 \mathrm{ml}$ and the BEV suspension ultracentrifuged $\left(150,000 \mathrm{~g}\right.$ at $4^{\circ} \mathrm{C}$ or $2 \mathrm{~h}$ in a Ti70 rotor (Beckman Instruments)). After ultracentfifugation, the supernatant was removed using a vacuum pump and the BEV pellets snap frozen in liquid nitrogen and stored at $-80^{\circ} \mathrm{C}$ prior to extraction.

\section{Nanoparticle analysis}

For BEVs generated in vitro (Fig. 2a) the size, concentration and zetapotential of the isolated Bt BEVs was determined using a ZetaView PMX-220 TWIN instrument according to manufacturer's instructions (Particle Metrix GmbH, Germany). Aliquots of BEV suspensions were diluted 1000- to 20,000-fold in particle-free PBS or water for analysis. Size distribution video data was acquired using the following settings: temperature: $25^{\circ} \mathrm{C}$; frames: 60 ; duration: 2 seconds; cycles: 2 ; positions: 11 ; camera sensitivity: 80 and shutter value: 100. Data were analysed using the ZetaView NTA software (version 8.05.12) with the following post acquisition settings: minimum brightness: 20; max area: 2000; min area: 5 and tracelength: 30 .

For BEVs generated in the mouse GIT, size distribution of vesicles was performed on $1 \mathrm{~mL}$ of BEV suspensions diluted 100-fold with PBS. Videos were generated using a Nanosight nanoparticle instrument (NanoSight Ltd, Malvern, USA) to count BEV numbers in BEV samples. A 1-min AVI file was recorded and analysed using NTA (Version 2.3 Build 0011 RC, Nanosight) software to calculate size distributions and vesicle concentrations using the following settings: Calibration: $166 \mathrm{~nm} /$ pixel; Blur auto: Detection threshold: 10, Minimum track length: auto, Temperature: $21.9 \mathrm{C}$, Viscosity: $0.96 \mathrm{cP}$. The accuracy of the measurement was confirmed using $100 \mathrm{~nm}$ silver nanoparticles (Sigma-Aldrich).

\section{Metabolites and enzyme enrichment in BEVs}

The enzymatic reactions inferred from the enzymes observed as being present in BEV were used to compile three sets of metabolites: substrate (but not product) metabolites, product (but not substrate) metabolites and those metabolites that were both substrates and products (involved in multiple reactions). These metabolites were measured in both media and metabolites were then ordered by fold-change in BEV with respect to $\mathrm{BHI}$.

\section{$B E V, E V$ and bacterial cell isolation from the mouse caecum}


Ten germfree C57BL/6 (males, 14 weeks old) mice were gavaged with $10^{8} \mathrm{CFU}$ Bt in $100 \mu \mathrm{L}$ PBS. Mice had unrestricted access to chow (Rat and Mouse $n^{\circ} 3$ breeding, Special Diet Services) and water for 2 days after which a group of 5 mice were deprived of food for 16 hours. The study was reviewed and approved by the Animal Welfare and Ethical Review Body (AWERB, University of East Anglia, Norwich, UK) and was conducted within the provisions of the Animals (Scientific Procedures) act 1986.

Post mortem, the caecal contents were collected and homogenised in PBS $(10 \% \mathrm{w} / \mathrm{v})$. Homogenates were centrifuged for $2 \mathrm{~min}$ at $100 \mathrm{~g}$ and the supernatant collected. A $100 \mu \mathrm{L}$ aliquot was removed to enumerate bacteria on BHI-haemin agar ( $=12 \pm 3 \times 10^{10} \mathrm{CFU} / \mathrm{g}$ colon content). The supernatants were then centrifuged at $5,500 \mathrm{~g}, 4^{\circ} \mathrm{C}$ for $15 \mathrm{~min}$. The cell pellets were rinsed twice with $30 \mathrm{~mL}$ PBS and snap frozen in liquid nitrogen and stored at $-80^{\circ} \mathrm{C}$ prior to extraction. The supernatants were filtered through polyethersulfone (PES) membranes (0.22 $\mu \mathrm{m}$ pore-size) (Sartorius). The sterility of the vesicles (BEV and EV)-containingfiltrate was confirmed by plating onto BHI-haemin agar. Vesicle suspensions were concentrated as described above. Following crossflow ultrafiltration, further purification of BEVs and EVs was performed by fractionation of the suspension [20] by size-exclusion chromatography using a CL2-B Sepharose (Sigma-Aldrich) $(120 \mathrm{~cm} \times 1 \mathrm{~cm}$ column) in PBS buffer. The absorbance of the fractions was measured at $280 \mathrm{~nm}$ and the first fractions corresponding to the first absorbance peak were pooled and concentrated to $1 \mathrm{~mL}$ with a Vivaspin 20 centrifugal concentrator (100 kDa molecular weight cut-off, Sartorius) and filtered through a $0.22 \mu \mathrm{m}$ PES membrane (Sartorius). Vesicle concentration was determined by Nanoparticle Tracking Analysis (NTA). The volume of the retentate was adjusted to $8.9 \mathrm{~mL}$ and the BEV suspension centrifuged $\left(150,000 \mathrm{~g}\right.$ at $4^{\circ} \mathrm{C}$ or $2 \mathrm{~h}$ in a Ti70 rotor (Beckman Instruments)). After centrifugation, the supernatant was removed using a vacuum pump and the vesicle pellets snap frozen in liquid nitrogen and stored at $-80^{\circ} \mathrm{C}$ prior to extraction.

\section{Proteomics}

Comparative proteomics was carried out on samples of BEVs produced in BHI versus BDM and from BEVs and EVs isolated from the caecum of fed or fasted mice. For the in vitro experiments vesicles were isolated (above) from 3 independent cultures for each culture medium. One of the samples obtained in BDM was excluded from further analysis as it produced anomalous results. In the comparison of BEVs generated in vivo 5 mice were used for each condition providing 10 datasets including ratios (fasted versus fed) for each protein identified with the level of confidence determined by the false discovery rate (FDR), that were then further analyzed. Parental cells were from BHI cultures or the caecum of Bt colonised mice (3 replicates for each condition). 
Samples for proteomics analysis consisted of $100 \mathrm{ug}$ of BEV or cell protein extract prepared and labelled at the Bristol University proteomics facility using TMT reagents (10-Plex format, Isobaric Mass Tagging kit, Thermo Scientific). Labelled samples were pooled and then fractionated using High $\mathrm{pH}$ Reverse Phase Liquid Chromatography. The resulting fractions were subjected to nano-LC MSMS using an Orbitrap Fusion Tribrid mass spectrometer with an SPS-MS3 acquisition method. Fragmentation of the isobaric tag released the low molecular mass reporter ions which were used to quantify the peptides. Protein quantitation was based on the median values of multiple peptides identified from the same protein, resulting in highly accurate protein quantitation between samples. The data sets were analysed using the Proteome Discoverer v2.1 software and run against the Bt VPI-5482 or mouse database and filtered with a 5\% (1\%) FDR cutoff.

\section{Proteomics data curation}

BEVs versus parent cells produced in vitro and in vivo: from 3092 listed proteins of the raw results to 2047. A hundred contaminant proteins (FALSE) were removed from the data. Using the $99 \%$ confidence level ( $<1 \%$ FDR), 213 additional proteins were removed. Proteins that were not found in BEVs (732) were also removed from the list resulting in a total of 2047 identified proteins. For the abundance ratio of BEV proteins (mouse caecum versus $\mathrm{BHI}$ ) those with a ratio $\geq 15$ and a PSMs $\geq 10$ were retained, excluding proteins that were not identified in the fasted versus fed animal experiment, resulting in a total of 102 proteins. To discriminate between proteins that are enriched in BEVs in vivo, the 36 proteins with an abundance ratio in the cell lysate (mouse caecum versus $\mathrm{BHI}$ ) $\geq 5$ were considered as non-enriched whereas the 66 proteins with an abundance ratio in cell lysates (mouse caecum versus BHI) $\leq 5$ were considered enriched in BEVs.

\section{Gene ontology analysis}

The proteins were categorized according to species specific gene ontology (GO) annotations using PANTHER version 14.0 at http://www.pantherdb.org/ [23].

\section{Electron microscopy}

Cells were grown in BHI to early stationary phase and visualised by negative staining electron microscopy. $2 \mu \mathrm{L}$ of liquid culture were applied to a 600-mesh copper TEM grid coated with formvar/carbon. The sample was left to settle out for 5 minutes and $2 \mu \mathrm{L}$ of $2 \mathrm{x}$ fixative ( $5 \%$ glutaraldehyde in $200 \mathrm{mM}$ sodium cacodylate buffer, $\mathrm{pH}$ 7.2) was added and left for 5 minutes. The grid was then immersed for 10 minutes in $10 \mu 1$ of $1 \mathrm{x}$ fixative, washed 5 times with $100 \mathrm{mM}$ sodium cacodylate buffer, $\mathrm{pH} 7.2$ and 5 times with ultrapure water $(1$ minute each). The grid was air dried before negative staining in $2 \%$ aqueous Uranyl 
acetate-stain was applied and removed immediately. Grids were air dried and viewed in a Jeol 1230 TEM operated at an accelerating voltage of $80 \mathrm{kV}$. Images were recorded on a Gatan One View 16MP digital camera.

Pellets of BEVs (including EVs for immunogold staining) were resuspended and fixed by vortex and pipetting in $100 \mu \mathrm{L} 2.5 \%$ Glutaraldehyde in 0.1M PIPES buffer. Large aggregates of material still present upon pellet resuspension, were removed by centrifugation for $2 \mathrm{~min}$ at low speed $(5 \mathrm{~g})$. A $50 \mu \mathrm{L}$ portion of supernatant was mixed 1:1 with cooled molten $4 \%$ low gelling temperature agarose (TypeVII, Sigma), solidified by chilling and cut into approximately $1 \mathrm{~mm}^{3}$ pieces. The BEV sample pieces were transferred into glass vials for further fixation in $2.5 \%$ glutaraldehyde in $0.1 \mathrm{M}$ PIPES buffer overnight at $4^{\circ} \mathrm{C}$. Fixed BEV sample pieces were washed in 0.1M PIPES buffer (3x) and post-fixed in $1 \% \mathrm{OsO}_{4}$ (Agar Scientific) for $2 \mathrm{~h}$. Following $\mathrm{OsO}_{4}$ fixation, samples were washed in deionised water (3x), followed by dehydration through an ethanol series $(30,50,70,90,3 \times 100 \%)$. The samples were infiltrated with a 1:1 mix of $100 \%$ ethanol to LR White medium grade resin, followed by a 1:2 and a 1:3 mix of $100 \%$ ethanol to LR White resin and finally $100 \%$ resin, with at least $1 \mathrm{~h}$ between changes. The resin was changed twice more with fresh $100 \%$ resin with periods of at least $8 \mathrm{~h}$ between changes. The sample pieces were each transferred into BEEM embedding capsules with fresh resin and polymerised for $24 \mathrm{~h}$ at $60^{\circ} \mathrm{C}$. Sections approximately $90 \mathrm{~nm}$ thick were cut using an ultramicrotome (Ultracut E, Reichert-Jung) with a glass knife, collected on $\mathrm{Cu}$ Formvar/carbon grids and stained sequentially with $2 \%$ uranyl acetate solution for $1 \mathrm{~h}$ at $21^{\circ} \mathrm{C}$, and $0.5 \%$ lead citrate solution for $1.5 \mathrm{~min}$ at $21^{\circ} \mathrm{C}$. Deionised water washes were performed $(5 \mathrm{x})$ following each of the staining steps. Sections were examined and imaged in a FEI Talos F200C transmission electron microscope at 200kV with a "Gatan One View" digital camera. For immunogold staining, a "short" version of the Aurion Immunogold labelling (IGL) protocol (http://www.aurion.nl/the aurion_method/Post_embedding_conv) was used with $1 \mathrm{~h}$ antibody incubations and detergent $(0.1 \%$ TWEEN). The primary antibody (anti-OmpA) was diluted $1 / 500$ and the secondary antibody (GAR-10, Agar Scientific, Stanstead, UK) was diluted 1/50. After antibody labelling, the sections were stained with $2 \%$ uranyl acetate for $40 \mathrm{~min}$. The sections were examined and imaged in a FEI Tecnai G2 20 Twin transmission electron microscope at $200 \mathrm{kV}$.

\section{Construction of a BT_2086 deletion mutant}

An 899 bp chromosomal DNA fragment upstream from BT_2086 and including the first 30 nucleotides of its 5 '-end region was amplified by PCR using the primer pair f-5'bsh1_SpHI, r-5'bsh1_SalI. This product was then cloned into the SpHI/SalI sites of the E. coli-Bacteroides suicide shuttle vector pGH014 [22]. A 900 bp chromosomal DNA fragment downstream from BT_2086, including the last 44 nucleotides of the 3'-end region, was amplified by PCR using the primer pair f-3'bsh1_BamHI, r-3'bsh1_SacI and was cloned 
into the BamHI/SacI sites of the pGH014-based plasmid. The resulting plasmid containing the $\Delta$ BT_2086::tetQ construct, was mobilized from E. coli strain GC10 into Bt by triparental filter mating [24], using E. coli HB101(pRK2013) as the helper strain. Transconjugants were selected on BHI-haemin agar containing gentamicin $(200 \mathrm{mg} / \mathrm{L})$ and tetracycline $(1 \mathrm{mg} / \mathrm{L})$. Determination of susceptibility to either tetracycline or erythromycin was done to identify recombinants that were tetracycline resistant and erythromycin susceptible after re-streaking transconjugant bacteria on LB-agar containing tetracycline or both antibiotics. PCR analysis and sequencing were used to confirm allelic exchange. A transconjugant, GH511 containing the $\Delta$ BT_2086::tetQ construct inserted into the Bt chromosome was selected for further studies.

\section{Bile salt hydrolase activity}

Thin layer chromatography to assess the activity and substrate specificity of BSHs in Bt were performed according to Sedláčková et al. [25]. Bt strains were grown in $5 \mathrm{~mL} \mathrm{BHI}$ for $16 \mathrm{~h}$. The cultures were centrifuged at $9000 \mathrm{~g}$ for $10 \mathrm{~min}$ at $4^{\circ} \mathrm{C}$, the cell pellets washed with $2 \mathrm{ml}$ of PBS and resuspended in $5 \mathrm{~mL}$ of PBS. $500 \mu \mathrm{L}$ of washed sample (or $5 \times 10^{9}$ BEVs in $500 \mu \mathrm{L}$ PBS) were mixed with $500 \mu \mathrm{L}$ of substrate solution (Na-GCA $0.3 \%$ and Na-TCA $0.3 \%$ in PBS) and incubated for $16 \mathrm{~h}$ at $37^{\circ} \mathrm{C}$. The TLC chamber containing the mobile phase (isoamyl acetate $40 \%$, propionic acid $30 \%$ and 1-propanol $20 \%$ in water) was equilibrated for 30 minutes. The reaction mixtures were speed-vacuum dried, the pellets dissolved in 500 $\mu \mathrm{L}$ of methanol and the solution was centrifuged at $14000 \mathrm{~g}$ at $4^{\circ} \mathrm{C}$ for $1 \mathrm{~min} .3 \mu \mathrm{L}$ of the supernatants and of the standards (cholic acid [CA], sodium taurocholic acid [Na-TCA] or sodium glycocholic acid [NaGCA] $5 \mathrm{mM}$ in methanol) were spotted onto silica gel plates (TLC Silica gel $60 \mathrm{~F}_{254}$, Merck). The plate was inserted into the chamber and allowed to run for about $40 \mathrm{~min}$ and removed when the solvent front was 1 to $2 \mathrm{~cm}$ from the top edges. The plate was dried at $110^{\circ} \mathrm{C}$ for $3 \mathrm{~min}$ and sprayed with a solution of phosphomolybdic acid $(10 \% \mathrm{w} / \mathrm{v}$ in ethanol). The plate was dried again until spots were visible.

\section{DPP4 assay}

DPP4 assays were performed as described by Beauvais et al. [26]. Briefly, $750 \mathrm{ul}$ of $50 \mathrm{mM}$ Tris $\mathrm{HCl}$ buffer (pH 7.5) and 50ul Ala-Pro-pNA (5 mg/mL in methanol) were added to $200 \mu \mathrm{l}$ of BEV suspension. The reaction mixture was incubated at $37^{\circ} \mathrm{C}$ and the $\mathrm{OD}_{405}$ was measured at $1 \mathrm{~min}$ intervals for $100 \mathrm{~min}$. The amount of protein in BEVs was determined using the Bio-Rad Protein Assay. 


\section{Results}

\section{Impact of nutrient availability on BEV biophysical characteristics and hydrolytic enzyme content}

Bt produces large amounts of uniform BEV particles which are released from the bacterial cell surface into the external milieu (Fig. 1). To test whether environmental factors have an impact on BEV structure, production and protein composition, Bt was cultured in either a complex (BHI) or defined and minimal (BDM) media and BEVs isolated from the culture supernatants. BEV concentration harvested from $\mathrm{Bt}$ grown in BHI and BDM media were similar while their average size increased from $135 \pm 6 \mathrm{~nm}$ in $\mathrm{BHI}$ to $205 \pm 3 \mathrm{~nm}$ in BDM (Fig. 2a). Electron microscopy imaging confirmed that BEVs from BHI and BDM were similar in appearance and structure although those produced in BDM were larger in size (Fig 2b). The average zeta potential of BEVs from both BHI and BDM media was $-25 \mathrm{mV}$ and $-22 \mathrm{mV}$ (in PBS, $\mathrm{pH} 7.2$, $25^{\circ} \mathrm{C}$ ) respectively, which is similar to what was reported for E. coli-derived BEVs [27].

The proteomic profile of BEVs produced in BHI versus BDM cultures were compared by differential proteomic analysis. In general, 1,438 proteins were identified corresponding to approximately $30 \%$ of the predicted proteome of parent cells [28]. Of note, the majority of proteins were more abundant in BEVs produced in nutrient-poor, BDM, medium (Fig. 3a). Proteins categorized according to universal gene ontology (GO) annotations showed that many of the proteins displaying an increase abundance (fold change $>3$ ) were hydrolases, and in particular glycoside hydrolases, and proteases in addition to transferases, oxidoreductases, ligases and lyases (Fig. 3b). Whereas the complete predicted proteome of Bt is composed of $64 \%$ acidic proteins ( $\mathrm{pI}<7.4$, physiological $\mathrm{pH}$ ), $79 \%$ of the BEV proteins were acidic, confirming the enrichment of acidic proteins in BEVs (data not shown).

The Bt transcriptome in response to nutrient availability was previously investigated by microarray analysis [29] using probe pairs derived from 4,779 predicted genes to compare transcriptional profiles obtained from $B t$ grown in rich versus minimal medium (with glucose as the sole carbon source) during early log phase to stationary phase. Accordingly, we selected the 250 most abundant proteins that were more, or less, abundant in the defined medium compared to the rich medium (highest peptide spectrum match PSM) with the level of expression of their corresponding genes determined under the same conditions (BDM-G). The results showed that the fold differences in protein and the corresponding RNA expression correlated with each other (Fig. 3c) (Spearman correlation coefficient $r_{s}=0.44, p=2.10^{13}$ ) indicating that BEV protein content reflected RNA levels in the parental cell. 
Our analysis also showed that there was a corresponding increase in the metabolites generated from reactions catalysed by the more abundant proteins present in BEVs produced in BDM (Table 1). Intriguingly, the concentration of the substrates specific for these enzymes [9] was also increased in BEVs indicating that some of the reactions are reversible and/or the diffusion of the substrate from the external milieu into BEVs is facilitated by the presence of higher levels of enzyme.

Since the BEV protein content was affected by nutrient availability in vitro, we investigated if nutrient deprivation in vivo (in fasting animals) could similarly lead to changes in the BEVs' proteome.

\section{BEV proteomic profile in vivo}

To assess whether nutrient deprivation affects the protein composition of BEVs produced by $\mathrm{Bt}$ in the GI tract, germfree mice were orally gavaged with $\mathrm{Bt}$ with one group of conventionalised mice allowed unrestricted access to food and water with a second group being deprived of food for $16 \mathrm{~h}$. BEVs extracted from the caecum were equivalent in size from both fed or fasted mice with a mean size of approximately $190 \mathrm{~nm}$ when measured with a NanoSight instrument (Fig. 4a). By contrast, 1.8 times more nanoparticles were recovered from the caecum of fasted mice compared to fed animals (Fig. 4a). The presence and identity of Bt BEVs in mouse caecal preparations was confirmed by immuno-EM using an antiserum specific for the outer membrane protein OmpA (BT_3852) of Bt (Fig. 4b).

Comparison of the proteome of caecal BEVs from fasted versus fed animals showed differences in protein abundance (Fig. 4c). However, they were less pronounced (between -6 and 3-fold) when comparing with in vitro cultures using BDM versus BHI media (Fig. 3a). Unlike for BEVs obtained in vitro, for which most of the proteins ( $82 \%$ ) displayed an increased abundance when produced in nutrient-poor growth medium, there were similar numbers of proteins exhibiting increased (48\%) and decreased (52\%) abundance, when comparing the two in vivo conditions (see results in Vesiclepedia, number).

Comparison of the proteome of caecal BEVs from fed and fasted animals showed differences in protein abundance (Fig. 4c). Of 558 proteins identified in BEVs extracted from fasted and fed mice, 322 were differentially abundant (fold change $\geq 1.3$ ). Of these, 142 were more abundant in BEVs extracted from fasting animals whereas 180 proteins were more abundantly represented in BEVs derived from fed animals. GSEA analysis [30] revealed two biological processes significantly enriched in BEVs from fasted animals, "peptide metabolic process" [GO:0006518] (70 proteins) and "protein processing" [GO:0016485] (69 proteins) gene ontologies. Sixty-nine of these proteins also belonged to the molecular function ontology "metallocarboxypeptidase activity" [GO:0004181]. The BEVs from fed animals contained a set of eight 
proteins displaying "serine-type peptidase" activity [GO:0008236] that were more abundant compared to fasted mice.

A large number of proteins expressed from polysaccharide utilization units (PULs), that are sets of neighbouring genes involved in the breakdown of specific glycans [31], were present in BEVs (Table S1) and were classified using the Polysaccharide-Utilization Loci DataBase (PULDB) http://www.cazy.org/PULDB/ [32] (Table S1). The starch degrading PUL66 was highly abundant which most likely reflects the high ( $\sim 34 \%)$ starch content of the animal chow. PULs involved in the degradation of rhamnogalacturonan-II (PUL77), pectic galactan (PUL86) and arabinogalactan (PUL65) were also highly abundant. Of note, in fasted mice there was an increased abundance of PULs capable of degrading host glycans and mucins (PULs 6, 19, 35, 37, 80 and 81).

\section{A set of proteins is selectively secreted in BEVs in the GI tract}

To investigate whether proteins are selectively enriched in BEVs in vivo, we first compared the proteome of BEVs harvested from the caecum of Bt mono-colonised germfree mice with that of BEVs generated in vitro in BHI media. A total of 102 proteins were identified based upon the abundance being at least 15 -fold higher in in vivo generated BEVs (Table S2, a and b). Next, we determined how many of these proteins might be enriched in BEVs in vivo as a result of their increased production in BEV's parental cells by comparing the levels of expression of the 102 proteins in caecal-derived parental cells with those grown in vitro in BHI medium (Table S2a, b). We assumed that for a given protein, the enrichment in BEVs in vivo occurs independently of protein expression, if the in vivo versus in vivo abundances of the protein in their parent cells are comparable, whereas its enrichment in BEVs is a consequence of higher expression in parental cells, if the abundance in the parent cells in vivo is increased compared to in vitro growth conditions.

This analysis revealed that the abundances of the majority of proteins (66/102) were comparable in parental cells generated in vivo or in vitro (Table S2a, Fig. 5a) excluding changes in protein production in parental cells contributing to the increased abundance of these proteins in BEVs in vivo. In contrast, 36 of the 102 proteins displayed a 5 -fold or higher abundance in parental cells under in vivo versus in vitro conditions (Table S2b, Fig. 5b) consistent with the increased production in parental cells contributing to their increased abundance in BEVs in vivo. To corroborate these findings, we compared the levels of expression of RNA for each of the 102 proteins enriched in BEVs in vivo using data obtained from a global transcriptomics analysis of Bt grown under different in vitro and in vivo conditions analogous to those we have used here [29]. Changes in the abundance of the 102 proteins in BEVs generated in vivo were closely mirrored by 
changes in RNA levels as the fold difference values between protein abundance in the cells and expression of the corresponding gene were significantly correlated [Spearman correlation coefficient $r_{s}=0.81(p<$ 0.0001)] (Fig. 5a and b, Table S2). These results are consistent with the selective enrichment of a set of (102) protein in BEVs in vivo that can occur in parallel with (36) or independently of (66) changes in protein production in parental cells.

The enrichment of the 102 proteins in BEVs generated in vivo was independent of food intake and availability since the abundance of these proteins was comparable in BEVs from fasted versus fed mice (fold change $\cong 1.0)$ (Fig. 5a, b).

We used the SignalP-5.0 Server software programme to predict the presence of known Gram-negative bacteria signal peptides amongst the 102 proteins enriched in BEVs (Fig. 5c). Most of the 36 proteins whose abundance values were 15 -fold or higher in BEVs in vivo versus in vitro were predicted to be transported via the bacterial Sec-dependent protein secretion system. By contrast, 31/66 of the proteins displaying a 5fold or less increase in abundance in parent cells were predicted to be secreted independently of known (Sec) bacterial secretion systems.

\section{Enrichment of bile salt hydrolases and dipeptidyl hydrolases IV in BEVs in vivo}

Prominent among the 36 proteins enriched in both BEVs and parent cells in vivo (Table S2) was the bile salt hydrolase (BSH) BT_2086. BSHs produced by gut commensal bacteria catalyse the hydrolysis of bile salts conjugated with the amino acids taurine or glycine residues and release free bile acids such as cholic acid (CA) and glycine and taurine [33]. Bt cells degrade both glyco- and tauro-conjugated bile acids GCA and TCA (Fig. 6). The specificity of the BSH encoded by BT_2086 was established by generating a Bt mutant lacking BT_2086 ( $\triangle$ BT2086). The mutant was unable to degrade TCA whereas hydrolysis of GCA to produce cholic acid was unaffected most likely reflecting the activity of the other predicted BSH, BT_1259 [28]. In the case of BEVs, levels of bile salt hydrolase activity and CA production were lower than that of parent cells as reflected in higher residual levels of GCA and TCA after incubation with BEVs (Fig. 6). Despite this it was clear that $\triangle \mathrm{BT} 2086$ generated BEVs produced strikingly less CA compared to BEVs from wild type Bt. These findings indicate that BEVs produced by Bt contain a BSH able to deconjugate tauro-conjugated bile salts.

The dipeptidyl-peptidase 4 (DPP4)-like protein (DPP6) encoded by BT_1314 was also abundant in BEVs in vivo (Table S2). Human DPP4 or CD26 truncates proteins containing the amino acid proline or alanine in the second position of the N-terminus, and DPP-4-like activity encoded by the intestinal microbiome has 
been proposed to constitute a novel mechanism to modulate protein digestion and host metabolism [34]. We tested therefore whether intact BEVs could hydrolyse DDP4 specific substrates (H-Ala-Pro-pnitroaniline) [26]. BEVs produced in vitro in BDM exhibited activity of $0.57 \mathrm{nmol} / \mathrm{min} / \mathrm{mg} \mathrm{BEV}$ total protein whereas BEVs isolated from BHI exhibited activity of $0.09 \mathrm{nmol} / \mathrm{min} / \mathrm{mg}$. This agrees with the abundance ratios measured for BT_1314 which was 6 times more abundant from BEVs obtained in BDM compared to those obtained in BHI. The same was also true for two other putative DPP4 enzymes detected in BEVs; BT_3254 (3 times more abundant from BDM) and BT_4193 (4 times more abundant from BDM). However, in vivo, BT_1314 was selectively enriched ( 20-fold) in BEVs.

\section{Proteome of EVs produced in the GI tract}

EVs in mammals are produced by almost all cell types and contribute to the coordinated signalling events and communication between the gut microbiota, intestinal epithelial cells, endothelial cells, and immune cells during homeostasis, immune activation, and inflammation [35]. Vesicles isolated from the caecum of fasted and fed mice mono-colonised with Bt consisted of a combination of BEVs and EVs. By running a peptide match against the UniProt mouse protein database, 1152 proteins from mouse EVs were identified (see results in Vesiclepedia, number). These included tetraspanin proteins which belong to a family of membrane proteins [36,37] including the cell surface glycoprotein CD9, six members of the 14-3-3 protein family comprising phospho-binding proteins, and nine annexins (Anxa1-7, Anxa11 and 13), all commonly found in eukaryotic vesicles [38]. From a comparison of our protein profile with that obtained from EVs of cultured human primary and metastatic colorectal cancer cells [38] (Source of human EV proteome: Vesiclepedia_500, at http://microvesicles.org/exp_summary?exp id=550) 333 (29\%) of the 1152 proteins overlapped and were present in both data sets. As expected, the EV cancer markers AXL, DNM2, CD59, CTNND1, EPHA2, ITGA1, ITGA5 and VIM $[39,40]$ were only present in human CRC EVs and were not detected in mouse caecal EVs.

We next compared the distribution of the overlapping proteins based on gene ontology (GO) categories (Table 2). Seven categories of proteins were dissimilarly represented in mouse caecal versus human cancer cell proteins. Proteins involved in developmental and cellular process, biological adhesion, and cellular component organisation (or biogenesis) were more frequent in human cancer cell EVs. Intriguingly, the proportion of proteins involved in cellular proliferation was increased in mouse caecal EVs. Furthermore, proteins contributing to multi-organism process and the immune system were also increased in caecal EVs. In comparing the abundance ratio for each protein contained in caecal EVs derived from fasted versus fed animals (Table 3), amongst EVs produced in fasted mice two serine protease inhibitors (A3M and A3K) 
were more abundant (5-fold and 2.7-fold, respectively). We also observed a 3.5 -fold increase in the abundance of the murine specific $\alpha$-defensin CRISC-2 in EVs produced in fasted mice.

\section{Discussion}

Our study provides new insights into microbe-host interactions in the mammalian GI tract and how BEVs can contribute to this crosstalk. Using the ubiquitous human commensal gut bacterium $\mathrm{Bt}$ as a model system, we have shown that the profile of proteins it packages into BEVs is influenced by nutrient availability, and provided evidence of the selective and exclusive enrichment of proteins in BEVs in vivo in the mouse GI tract that include enzymes capable of influencing host metabolism.

From previous work on bacterial pathogens it is known that bacterial proteins including virulence factors are selectively enriched in BEVs, consistent with vesiculation being a coordinated rather than passive process $[5,41,42]$. Virulence factors enriched in BEVs include gingipain proteases produced by the human oral pathogen Porphyromonas gingivalis, or the virulence factors VacA, urease and CagA produced by the gastric pathogen Helicobacter pylori, whereas other abundant cellular proteins not contributing to infection are excluded from BEVs $[43,44]$. Like for pathogens, proteomic analysis of BEVs produced by cultured commensal Bacteroides species identified proteins found exclusively in BEVs, including acidic lipoproteins with hydrolytic and carbohydrate-binding activities encoded by PULs $[15,45]$. Our analysis of BEV proteins under different culture conditions highlights the ability of $\mathrm{Bt}$ to effectively respond to nutrient stress by changing the profile of proteins it produces and packages in its BEVS, as predicted in a prior transcriptomics based study [29]. A similar phenomenon has been described in Campylobacter jejuni, which, although considered to be a commensal bacterium in avian hosts, is pathogenic and causes bacterial gastroenteritis in humans [46]. Proteomic analysis of the $C$. jejuni BEVs identified numerous proteins with differential abundance under culture conditions reflecting the different body temperatures of the two hosts, with significantly higher amounts of virulence proteins associated with BEVs from cultures at $37^{\circ} \mathrm{C}$ culture compared to BEVs produced at $42^{\circ} \mathrm{C}$ [47].

Our analysis of BEVs produced in vivo reveals that a set of cellular proteins (66) are selectively enriched in BEVs compared to their parental cells. In addition, as the levels of these proteins were comparable in fed versus fasted animals the process responsible for the accumulation of these proteins into BEVs functions independently of nutrient (food) supply (Fig. 5a). Thus, local environmental factors other than diet and nutrient supply are involved in the selection and secretion of a set of proteins into BEVs. Furthermore, based upon the known functionality of some of these proteins (e.g. dipeptidyl-peptidase and asparaginase) they are most likely selectively packaged into BEVs by the bacterium with the purpose of influencing host 
cell physiology and in particular, metabolism. The mechanisms that account for this enrichment of proteins in BEVs is unknown and likely to involve unique processes as nearly half of the proteins enriched in BEVs are not predicted to be secreted by a known bacterial secretion system.

The dipeptidyl-peptidase encoded by BT_1314 is enriched in BEVs in vivo and has the potential to influence host physiology via its effect on protein and glycan (e.g. gluten) digestion, signal transduction and apoptosis [34]. Based upon its ability to cleave and inactivate various signalling molecules important in metabolism (i.e. incretins), the immune system (i.e. growth factors and cytokines) and CNS (i.e. neuropeptides) [48-50] it is tempting to speculate that upon accessing the systemic circulation [7] BEVs can impact on various aspects of host physiology and behaviour, a possibility that awaits confirmation from further studies. The type II L-asparaginase encoded by BT_2757 was also selectively enriched in BEVs in vivo. Asparaginase activity is required to deamidate asparagine to aspartate, an essential amino acid for proliferating mammalian cells (e.g. cancer cells) and as a neurotransmitter [51,52]. The human asparaginase enzyme (ASPG) exhibits a relatively low affinity for L-asparagine while bacterial enzymes, that are commonly used as anticancer drugs, have a higher affinity for the substrate [53]. Indeed, E. coli-derived asparaginase is used in food manufacturing to reduce levels of the human carcinogen acrylamide [54] and clinically to treat leukemia and lymphoma patients [55]. The uptake of aspartate generated from asparaginase cleavage of asparagine is inefficient in most mammalian cells [51]. It can therefore be envisaged that following internalization of Bt BEVs into the cytosol of mammalian cells such as intestinal epithelial cells [7], BEVs could supply cells with asparaginase activity and address a shortage of aspartate to aid host cell metabolism. Our findings and that of Yao and colleagues [56] demonstrating that Bt BEVS produced in the mouse GI tract contain abundant quantities of biologically active, BT_2086-encoded, bile salt hydrolase is of potential significance for host physiology. Bile acid signaling pathways mediate insulin-resistance, obesity, lipid metabolism and systemic metabolic processes [33].

Fasting results in an increase in the relative abundance of members of the Bacteroidetes phylum irrespective of the fasting period (1-3 days) [57]. This increase can be explained by the ability of some phyla members to utilize host glycans in the absence of dietary equivalent glycans $[15,58,59]$, which was also observed in fasting mice that are a model for multiple sclerosis [60]. These observations indicate that the survival and growth of Bt may not be adversely affected by a lack of dietary nutrients in the GI tract [57]. This may explain the small variations observed in the proteome of BEVs produced from Bt colonising the lower GI tract of fasted mice when compared to the dramatic changes observed in the proteome of BEVs produced in the low nutrient culture medium BDM. Furthermore, we did not observe an increase in host glycan- 
specific and surface-exposed glycohydrolases in BEVs from fasted animals. Indeed, their abundance exhibited a downward trend (up to 2-fold) compared to their levels in BEVs from fed animals.

It is interesting to note that for all PULs the abundance of the integral membrane oligosaccharide importer SusC is increased by about fifty percent in BEVs produced under fasting conditions whereas for the other proteins belonging to the same PULs, including SusD (nutrient binding accessory protein) they are equally abundant or less abundant under fasting versus fed conditions. The glycosyl hydrolases associated to PUL systems which are preferentially packaged into BEVs [45] can provide substrates to support the growth of other bacteria in animals harbouring a conventional microbiota, conferring a "public good" function to BEVs $[13,61]$.

As part of this study we established the proteome profile of mammalian EVs in the mouse intestine. In comparing the abundance ratio for each protein contained in caecal EVs derived from fasted versus fed animals (Table 3), EVs produced in fasted mice contained two serine protease inhibitors (A3M and A3K serpins) with their abundance increased 5-fold and 2.7-fold, respectively. Moreover, 7 additional serpins (protease inhibition activity, InterPro family IPR000215) were identified with similar abundance in fasted and fed mice. It has been reported that high protease activity measured in the feces of patients suffering from irritable bowel syndrome correlates with a decrease in microbial diversity [62]. It is therefore tempting to speculate that the various serine protease inhibitors detected and identified in EVs produced in mice mono-colonised with Bt (submitted to Vesiclepedia, number) is a consequence of the lack of microbial diversity, and is to counteract the detrimental effect of proteases present in high abundance in the gut lumen [62] of mono-colonised mice.

We also compared the protein profile of mouse caecal EVs with that obtained from EVs of cultured human primary and metastatic colorectal cancer cells [38]. Human and mouse small intestines share many similarities in their intestinal microbial defence strategies, including production of $\alpha$-defensins which are also found in EVs [35]. Mice, however, produce a unique antimicrobial peptide and member of the CRS (cryptdin-related sequences)-peptide family, not found in man [63]. We observed a 3.5-fold increase in the abundance of the CRISC-2 $\alpha$-defensins in EVs produced in fasted mice. Whether a decrease in nutrient availability in the mouse intestine leads to increased expression of CRISC-2, to an increased number of the secretory Paneth cells and/or to CRISC-2 preferentially packaged into EVs still needs to be determined.

In summary our findings provide evidence for the influence of unfavourable growth conditions on BEV protein composition, and for the selective and exclusive enrichment of proteins in BEVs in vivo in the 
mouse GI tract that include enzymes capable of influencing the host metabolism. Furthermore, other

2 proteins enriched in BEVS in vivo are translocated more abundantly into vesicles because of higher

3 expression in their parent cells. Further investigations are needed to evaluate the impact of selected

4 candidates such as BSH, DPP4-like dipeptidyl-peptidase or asparaginase on host physiology. This will help

5 further in defining determinants in BEV-host interactions playing key roles in the maintenance of intestinal

6 metabolism and homeostasis.

7

8 Supplemental online material

$9 \quad$ Table S1 and Table S2.

10

\section{Acknowledgments}

We thank Jake Richardson from the JIC Bioimaging facility for his contribution to this publication. We also thank Dr Rokas Juodeikis for contributing to generation of the EM imaging and Dr Dimitris Latousakis for his help with TLC.

Disclosure statement

No potential conflict of interest was reported by the author.

\section{Funding}

This work was supported in part by the UK Biotechnology and Biological Sciences Research Council (BBSRC) under grant numbers BB/J004529/1, BB/R012490/1, and BBS/E/F000PR10355 (SC). 


\section{REFERENCES}

1. Sandrini $S$, Aldriwesh $M$, Alruways $M$, et al. Microbial endocrinology: host-bacteria communication within the gut microbiome. J. Endocrinol. 2015;225:R21-34.

2. Keely SJ. Decoding host-microbiota communication in the gut - now we're flying! J. Physiol. 2017;595:417-418.

3. Tulkens J, De Wever O, Hendrix A. Analyzing bacterial extracellular vesicles in human body fluids by orthogonal biophysical separation and biochemical characterization. Nat. Protoc. 2020;15:40-67.

4. Olsen I, Amano A. Outer membrane vesicles - offensive weapons or good Samaritans? J. Oral. Microbiol. 2015;7:27468.

5. Cecil JD, Sirisaengtaksin N, et al. (2019) Outer Membrane Vesicle-Host Cell Interactions. Microbiol. Spectr. 2019;7:10.1128/microbiolspec.

6. Caruana JC, Walper SA. Bacterial Membrane Vesicles as Mediators of Microbe - Microbe and Microbe - Host Community Interactions. Front. Microbiol. 2020;11:432.

7. Jones EJ, Booth C, Fonseca S, et al. The uptake, trafficking, and biodistribution of Bacteroides thetaiotaomicron generated outer membrane vesicles. Front. Microbiol. 2020;11:57.

8. Guerrero-Mandujano A, Hernández-Cortez C, Ibarra JA, et al. The outer membrane vesicles: Secretion system type zero. Traffic 2017;18:425-432.

9. Bryant WA, Stentz R, Le Gall G, et al. In silico analysis of the small molecule content of outer membrane vesicles produced by Bacteroides thetaiotaomicron indicates an extensive metabolic link between microbe and host. Answers to naysayers regarding microbial extracellular vesicles Front. Microbiol. 2017;8:2440.

10. Pérez-Cruz C, Carrion O, Delgado L, et al. A new type of outer membrane vesicles produced by the Gram-negative bacterium Shewanella vesiculosa M7T: implications for DNA content. Appl. Environ. Microbiol. 2013;79:1874-1881.

11. Perez-Cruz C, Delgado L, Lopez-Iglesias C, et al. Outer-inner membrane vesicles naturally secreted by gram-negative pathogenic bacteria PLoS ONE, 2015;10:e011689.

12. Gill S, Catchpole R, Forterre P. Extracellular membrane vesicles in the three domains of life and beyond. FEMS Microbiol. Rev. 2019;43:273-303.

13. Rakoff-Nahoum S, Coyne MJ, Comstock LE. An ecological network of polysaccharide utilization among human intestinal symbionts. Curr. Biol. 2014;24:40-49.

14. Stentz R, Carvalho AL, Jones E, et al. Fantastic voyage: the journey of intestinal microbiotaderived microvesicles through the body. Biochem. Soc. Trans. 2018;46:1021-1027. 
15. Elhenawy W, Debelyy MO, Feldman MF. Preferential packing of acidic glycosidases and proteases into Bacteroides outer membrane vesicles. mBio 2014;5:e00909-14.

16. Stentz R, Osborne S, Horn N, et al. A bacterial homolog of a eukaryotic inositol phosphate signaling enzyme mediates cross-kingdom dialog in the mammalian gut. Cell. Rep. 2014;6:646656.

17. Shen Y, Giardino Torchia ML, et al. Outer membrane vesicles of a human commensal mediate immune regulation and disease protection. Cell Host Microbe 2012;12:509-520.

18. Hickey CA, Kuhn KA, Donermeyer DL, et al. Colitogenic Bacteroides thetaiotaomicron antigens access host immune cells in a sulfatase-dependent manner via outer membrane vesicles. Cell Host Microbe 2015;17:672-680.

19. Kaparakis-Liaskos M, Ferrero R. Immune modulation by bacterial outer membrane vesicles. Nat. Rev. Immunol. 2015;15:375-387.

20. Durant L, Stentz R, Noble A, et al. Bacteroides thetaiotaomicron-derived outer membrane vesicles promote regulatory dendritic cell responses in health but not in inflammatory bowel disease. Microbiome 2020;8:88.

21. Toyofuku M, Nomura N, Eberl L. Types and origins of bacterial membrane vesicles. Nat. Rev. Microbiol. 2018;13:1.

22. Stentz R, Horn N, Cross K, et al. Cephalosporinases associated with outer membrane vesicles released by Bacteroides sp. protect gut pathogens and commensals against $\beta$-lactam antibiotics. J. Antimicrob. Chemother. 2015;70:701-709.

23. Mi H, Muruganujan A, Huang X, et al. Protocol Update for large-scale genome and gene function analysis with the PANTHER classification system (v.14.0). Nat. Protoc. 2019;14:703-721.

24. Shoemaker NB, Getty C, Gardner JF, et al. Tn 4351 transposes in Bacteroides spp and mediates the integration of plasmid R751 into the Bacteroides chromosome. J. Bacteriol. 1986;165:929936.

25. Sedláčková $\mathrm{P}$, Horáčková $\breve{\mathrm{S}}$, Shi $\mathrm{T}$, et al. Two different methods for screening of bile salt hydrolase activity in Lactobacillus strains. Czech J. Food Sci. 2015;33:13-18.

26. Beauvais A, Monod M, Wyniger J, et al. Dipeptidyl-peptidase IV secreted by Aspergillus fumigatus, a fungus pathogenic to humans. Infect. Immun. 1997;65:3042-3047.

27. Gnopo YMD, Misra A, Hsu HL, et al. Induced fusion and aggregation of bacterial outer membrane vesicles: Experimental and theoretical analysis. J. Colloid Interface Sci. 2020;578:522-532.

28. Xu J, Bjursell MK, Himrod J, et al. A genomic view of the human-Bacteroides thetaiotaomicron symbiosis. Science 2003;299:2074-2076. 
29. Sonnenburg JL, Xu J, Leip DD, et al. Glycan foraging in vivo by an intestine-adapted bacterial symbiont. Science. 2005;307:1955-1959.

30. Subramanian A, Tamayo P, Mootha VK, et al. Gene set enrichment analysis: a knowledge-based approach for interpreting genome-wide expression profiles. Proc. Natl. Acad. Sci. USA. 2005;102:15545-15550.

31. Terrapon N, Lombard V, Gilbert HJ, et al. Automatic prediction of polysaccharide utilization loci in Bacteroidetes species. Bioinformatics 2015; 31:647-655.

32. Terrapon N, Lombard V, Drula É, et al. PULDB: the expanded database of Polysaccharide Utilization Loci. Nucleic Acids Res. 46: D677-D683.

33. Taylor SA, Green RM. Bile acids, microbiota, and metabolism. Hepatology. 2018;68:1229-1231.

34. Olivares M, Schüppel V, Hassan AM, et al. The potential role of the dipeptidyl peptidase-4-like activity from the gut microbiota on the host health. Front. Microbiol. 2018;9:1900.

35. Bui TM, Mascarenhas LA, Sumagin R. Extracellular vesicles regulate immune responses and cellular function in intestinal inflammation and repair. Tissue Barriers 2018;6:e1431038.

36. Raposo G, Stoorvogel W. Extracellular vesicles: exosomes, microvesicles, and friends. J Cell Biol. 2013;200:373-383.

37. Hessvik NP, Llorente A. Current knowledge on exosome biogenesis and release. Cell. Mol. Life Sci. 2018;75:193-208.

38. Choi DS, Choi DY, Hong BS, et al. Quantitative proteomics of extracellular vesicles derived from human primary and metastatic colorectal cancer cells. J. Extracell. Vesicles. 2012;1:10.3402/jev.v1i0.18704.

39. Rontogianni S, Synadaki E, Li B, et al. Proteomic profiling of extracellular vesicles allows for human breast cancer subtyping. Commun. Biol. 2019;2:325.

40. Kosaka, N, Kogure, A, Yamamoto, $\mathrm{T}$ et al. Exploiting the message from cancer: the diagnostic value of extracellular vesicles for clinical applications. Exp. Mol. Med. 2019;51:31.

41. Veith PD, Chen YY, Gorasia DG, et al. Porphyromonas gingivalis outer membrane vesicles exclusively contain outer membrane and periplasmic proteins and carry a cargo enriched with virulence factors. J. Proteome Res. 2014;13:2420-2432.

42. Friedrich V, Gruber C, Nimeth I, et al. Outer membrane vesicles of Tannerella forsythia: biogenesis, composition, and virulence. Mol. Oral Microbiol. 2015;30:451-473.

43. Haurat MF, Aduse-Opoku J, Rangarajan M, et al. Selective sorting of cargo proteins into bacterial membrane vesicles. J. Biol. Chem. 2011;286:1269-1276. 
44. Zavan L, Bitto NJ, Johnston EL, et al. Helicobacter pylori growth stage determines the size, protein composition, and preferential cargo packaging of outer membrane vesicles. Proteomics 2019;19:e1800209.

45. Valguarnera E, Scott NE, Azimzadeh P, et al. Surface exposure and packing of lipoproteins into outer membrane vesicles are coupled processes in Bacteroides. mSphere 2018;3:e0559-18.

46. Rukambile E, Sintchenko V, Muscatello G, et al. Infection, colonization and shedding of Campylobacter and Salmonella in animals and their contribution to human disease: A review. Zoonoses Public Health. 2019;66:562-578.

47. Taheri N, Fällman M, Wai $\mathrm{SN}$, et al. Accumulation of virulence-associated proteins in Campylobacter jejuni outer membrane vesicles at human body temperature. J. Proteomics 2019;195, 33-40.

48. Barnett A. DPP-4 inhibitors and their potential role in the management of type 2 diabetes. Int. J. Clin. Pract. 2006;60:1454-1470.

49. Mentlein R. Dipeptidyl-peptidase IV (CD26)-role in the inactivation of regulatory peptides. Regul. Pept. 1999;85:9-24.

50. Chen X. Biochemical properties of recombinant prolyl dipeptidases DPP-IV and DPP8. Adv. Exp. Med. Biol. 2006;575:27-32.

51. Sullivan LB, Luengo A, Danai LV, et al. Aspartate is an endogenous metabolic limitation for tumour growth. Nat. Cell. Biol. 2018;20:782-788.

52. D'Aniello S, Somorjai I, Garcia-Fernàndez J, et al. D-Aspartic acid is a novel endogenous neurotransmitter. FASEB J. 2011;25:1014-1027.

53. Belviso $\mathrm{S}$, Iuliano R, Amato R, et al. The human asparaginase enzyme (ASPG) inhibits growth in leukemic cells. PloS one, 2017;12:e0178174.

54. Xu F, Oruna-Concha M-J, Elmore JS. The use of asparaginase to reduce acrylamide levels in cooked food. Food Chemistry 2016;210:163-171.

55. Egler RA, Ahuja SP, Matloub Y. L-asparaginase in the treatment of patients with acute lymphoblastic leukemia. J. Pharmacol. Pharmacother. 2016;7:62-71.

56. Yao L, Seaton SC, Ndousse-Fetter $\mathrm{S}$, et al. A selective gut bacterial bile salt hydrolase alters host metabolism. Elife 2018;7:e37182.

57. Kohl KD, Amaya J, Passement CA, et al. Unique and shared responses of the gut microbiota to prolonged fasting: A comparative study across five classes of vertebrate hosts. FEMS Microbiology Ecology, 2014;90:883-894.

58. Macfarlane GT, Gibson GR. Formation of glycoprotein degrading enzymes by Bacteroides fragilis. FEMS Microbiol. Lett. 1991;61:289-293. 
59. Martens EC, Chiang HC, Gordon JI. Mucosal glycan foraging enhances fitness and transmission of a saccharolytic human gut bacterial symbiont. Cell Host Microbe. 2008;4:447-457.

60. Cignarella F, Cantoni C, Ghezzi L, et al. Intermittent fasting confers protection in CNS autoimmunity by altering the gut microbiota. Cell. Metab. 2018;27:1222-1235.e6.

61. Rakoff-Nahoum S, Foster KR, Comstock LE. The evolution of cooperation within the gut microbiota. Nature 2016;533:255-259.

62. Edogawa S, Edwinson AL, Peters S, et al. Serine proteases as luminal mediators of intestinal barrier dysfunction and symptom severity in IBS. Gut 2019;69:62-73.

63. Andersson ML, Karlsson-Sjöberg JM, Pütsep KL. CRS-peptides: unique defense peptides of mouse Paneth cells. Mucosal. Immunol. 2012;5:367-376. 


\section{Figure Legends}

Figure 1. Release of BEVs from the cell surface of Bt into the external milieu. The cells were grown in BHI to early stationary phase and visualised by negative staining electron microscopy.

Figure 2. Impact of nutrient availability on BEV structure (a) Nanoparticle tracking analysis of BEVs suspensions obtained from Bt grown in nutrient-rich (BHI) or nutrientpoor (BDM) culture media ( $\mathrm{n}=3$ ). (b) EM images of BEVs derived from Bt cells grown in complex medium BHI or defined medium BDM.

Figure 3. Proteomic profiles of BEVs produced in nutrient-rich and nutrient-poor media (a) Abundance ratio of each of the 1,438 proteins identified in protein profiling of BEVs obtained in BDM versus BHI. Proteins with a ratio higher than 1 are marked in red and those with a ratio below 1 are indicated in blue. (b) Proteins displaying an increased abundance (fold change $>3$ ) were categorized according to universal gene ontology (GO) annotations: Blue bars represent the total number of protein representing each category in BEVs: Orange bars represent the number of proteins of each category in BEVs that are $>3$ fold more abundant in BDM vs BHI. (c) Impact of growth medium on BEV proteome and parent cell transcriptome. Correlation between the abundance ratios of each of the 250 most abundant proteins (highest peptide spectrum match PSM) and the level of expression of the corresponding gene (Sonnenburg et al., 2018) identified in rich (BHI) versus minimal defined (BDM) growth media.

Figure 4. Structure and protein composition of BEVs produced in the mouse GIT. (a) Size distribution of extracellular vesicles produced in the caecum of germfree mice mono-colonised with Bt. The vesicles were extracted from caecal contents of mice either fed ad libitum or fasted for $16 \mathrm{~h}$ ( $\mathrm{n}=5$ ea.). (b) TEM images of vesicles extracted from the caecum of fed or fasted mice. Lower panel shows immunodetection of $\mathrm{Bt}$ BEVs from fed mice using an in-house generated rabbit anti-Bt OmpA antiserum and colloidal gold antirabbit Ig. Scale bar $=100 \mathrm{~nm}$. (c) Comparison of the abundance of each of the 558 proteins identified in BEVs extracted from fasted versus fed mice. Proteins with a ratio higher than 1 are marked in red and those with a ratio below 1 are indicated in blue.

Figure 5. Proteins enriched in BEVs produced in the mouse GIT. The 102 proteins found to be enriched in BEVs in vivo (fold change $\geq 15$, Table S2), were divided into two groups based upon comparing their levels in BEVs versus parental cells. (a) 66 proteins had a less than 5-fold increase in abundance in the parent cells in vivo (Table S2) are combined in (a), and results of the 36 proteins with a greater than 5-fold change in the parent cells in vivo (Table S2) are combined in (b). For the two groups of proteins in (a) and (b), their expression is compared to that of the mRNA expression level of the corresponding gene in cells grown in 
1 similar conditions [29]. The impact of food withdrawal (Fasted/Fed) on the abundance of proteins in the

2 two groups is also shown. (c) SignalP-5.0 Server at http://www.cbs.dtu.dk/services/SignalP/ was used to 3 predict the presence of different types of signal peptides present amongst the two sets of enriched proteins.

4 The 66 set is represented by dark blue bars and the 36 set of enriched proteins is represented by light blue 5 bars. Sec/SPI are secretory signal peptides transported by the Sec translocon and cleaved by Signal 6 Peptidase I; Sec/SPII are lipoprotein signal peptides transported by the Sec translocon and cleaved by Signal 7 Peptidase II; Tat/SPI are Tat signal peptides transported by the Tat translocon and cleaved by Signal 8 Peptidase I; "Other" are predicted secreted proteins translocated by uncharacterized secretion pathways. 9

Figure 6. Bile salt hydrolase activity in Bt and BEVs. Thin layer chromatography was used to confirm BSH activity and substrate specificity of the BSH encoded by BT_2086 present in Bt cells and BEVs obtained 12 after growth in BHI. Cholic acid [CA], taurocholic acid [TCA] and glycocholic acid [GCA] standards were 13 incubated with whole cells or BEVs from wild type (WT) or a Bt BSH1 deletion mutant ( $\triangle \mathrm{BT} \_2086$ ) for $1424 \mathrm{~h}$ at $37^{\circ} \mathrm{C}$ after which supernatants were spotted onto a silica gel plates. The plate was inserted into a TLC chamber, run for $40 \mathrm{~min}$ and stained with phosphomolybdic acid. 
Table 1 Enrichment of metabolites produced or utilised by the reactions catalysed by enzymes with increased abundance in BEV produced in BDMs versus BHI.

\begin{tabular}{llll}
\hline Criterion & Products & Substrates & Both \\
\hline Fold-change $>=\mathbf{5}^{*}$ & $1.5 \times 10^{-6}$ & $3.0 \times 10^{-1}$ & $1.5 \times 10^{-5}$ \\
\hline $\mathbf{q}<=\mathbf{0 . 0 5}($ FDR) $* *$ & $1.1 \times 10^{-11}$ & $5.9 \times 10^{-7}$ & $2.3 \times 10^{-4}$ \\
\hline
\end{tabular}

* P-values were calculated using the Gene Set Enrichment Analysis (GSEA) algorithm ** The BenjaminiHochberg correction was used to account for multiple testing (three sets, as defined above), giving q-values at a 0.05 false discovery rate (FDR) level. 
Table 2 Comparison of mouse caecal and human cancer cell EV gene ontology

\begin{tabular}{|c|c|c|c|c|c|c|}
\hline \multirow{2}{*}{$\begin{array}{l}\text { EV proteome } \\
\text { PANTHER GO-Slim Biological Process }\end{array}$} & \multicolumn{3}{|c|}{ Mouse } & \multicolumn{3}{|c|}{ Human } \\
\hline & $\begin{array}{l}\text { Number } \\
\text { of } \\
\text { proteins* }\end{array}$ & $\begin{array}{c}\% \\
\text { proteins } \\
\text { vs total }\end{array}$ & $\begin{array}{l}\% \\
\text { proteins } \\
\text { vs class } \\
\text { hits }\end{array}$ & $\begin{array}{c}\text { Number } \\
\text { of } \\
\text { proteins* }\end{array}$ & $\begin{array}{c}\% \\
\text { proteins } \\
\text { vs total }\end{array}$ & $\begin{array}{l}\% \\
\text { proteins } \\
\text { vs class } \\
\text { hits }\end{array}$ \\
\hline Developmental process (GO:0032502) & 47 & 4.50 & 2.70 & 70 & 6.70 & 3.70 \\
\hline Multicellular organismal process (GO:0032501) & 43 & 4.10 & 2.50 & 54 & 5.20 & 2.90 \\
\hline Cellular process (GO:0009987) & 410 & 39.50 & 23.90 & 512 & 49.10 & 27.10 \\
\hline Reproduction (GO:0000003) & 5 & 0.50 & 0.30 & 4 & 0.40 & 0.20 \\
\hline Cell population proliferation (GO:0008283) & 12 & 1.20 & 0.70 & 6 & 0.60 & 0.30 \\
\hline Localization (GO:0051179) & 170 & 16.40 & 9.90 & 157 & 15.10 & 8.30 \\
\hline Reproductive process (GO:0022414) & 5 & 0.50 & 0.30 & 4 & 0.40 & 0.20 \\
\hline Multi-organism process (GO:0051704) & 17 & 1.60 & 1.00 & 4 & 0.40 & 0.20 \\
\hline Biological adhesion (GO:0022610) & 16 & 1.50 & 0.90 & 26 & 2.50 & 1.40 \\
\hline Immune system process (GO:0002376) & 37 & 3.60 & 2.20 & 10 & 1.00 & 0.50 \\
\hline Cellular component organization or biogenesis (GO:0071840) & 131 & 12.60 & 7.70 & 213 & 20.40 & 11.30 \\
\hline Biological regulation (GO:0065007) & 230 & 22.20 & 13.40 & 228 & 21.90 & 12.10 \\
\hline Growth (GO:0040007) & 3 & 0.30 & 0.20 & 5 & 0.50 & 0.30 \\
\hline Signaling (GO:0023052) & 111 & 10.70 & 6.50 & 107 & 10.30 & 5.70 \\
\hline Metabolic process (GO:0008152) & 261 & 25.20 & 15.20 & 294 & 28.20 & 15.60 \\
\hline Pigmentation (GO:0043473) & 2 & 0.20 & 0.10 & 2 & 0.00 & 0.00 \\
\hline Response to stimulus (GO:0050896) & 175 & 16.90 & 10.20 & 151 & 14.50 & 8.00 \\
\hline Rhythmic process (GO:0048511) & 2 & 0.20 & 0.10 & 1 & 0.10 & 0.10 \\
\hline Locomotion (GO:0040011) & 35 & 3.40 & 2.00 & 40 & 3.80 & 2.10 \\
\hline
\end{tabular}

*The number of proteins from a category noticeably increased or decreased is indicated in red and blue, respectively 
Table 3 Differently abundant proteins in EVs from the caecum of fasted versus fed mice

\begin{tabular}{|c|c|c|c|c|}
\hline Accession & Description & Ratio: (S) / (NS)* & T-test & Protein Class \\
\hline \multicolumn{5}{|c|}{ Increased abundance in fasted mice } \\
\hline Q03734 & Serine protease inhibitor A3M & 5.01 & 0.013575 & Protease inhibitor \\
\hline Q5ERJ0 & CRS1C-2 alpha-defensin & 3.47 & 0.007428 & Defensin \\
\hline Q9CPY7 & Cytosol aminopeptidase Lap3 & 2.79 & 0.047037 & Aminopeptidase \\
\hline A0A0R4J0I1 & Serine protease inhibitor A3K & 2.682 & 0.03133 & Protease inhibitor \\
\hline Q00898 & Alpha-1-antitrypsin 1-5 & 2.117 & 0.010164 & Protease inhibitor \\
\hline Q9CYL5 & Golgi-associated plant pathogenesis-related protein 1 & 2.014 & 0.022046 & - \\
\hline Q8R000 & Organic solute transporter subunit alpha & 2.01 & 0.017694 & Transport \\
\hline \multicolumn{5}{|c|}{ Decreased abundance in fasted mice } \\
\hline E9Q7Q0 & Mucin-4 & 0.499 & 0.001219 & $\begin{array}{l}\text { Cell-matrix } \\
\text { adhesion }\end{array}$ \\
\hline I6L958 & Igk protein & 0.498 & 0.023509 & Immunoglobulin \\
\hline P02816 & Prolactin-inducible protein homolog & 0.489 & 0.01436 & - \\
\hline E9Q035 & Uncharacterized protein & 0.47 & 0.012476 & Transport/Carrier \\
\hline B1AWC9 & Phosphodiesterase & 0.463 & 0.033889 & Phosphodiesterase \\
\hline Q7TQD7 & Myolb protein & 0.46 & 0.030377 & Actin-binding \\
\hline B2RS76 & Carboxypeptidase B1 (Tissue) & 0.437 & $2.29 \mathrm{E}-05$ & Peptidase \\
\hline Q9CQC2 & Colipase & 0.437 & 0.001193 & $\begin{array}{l}\text { Protein-binding } \\
\text { activity modulator }\end{array}$ \\
\hline Q9D2R0 & Acetoacetyl-CoA synthetase & 0.434 & 0.007679 & Ligase \\
\hline Q64444 & Carbonic anhydrase 4 & 0.422 & 0.000349 & Lyase \\
\hline Q4FJZ7 & Ada protein & 0.41 & 0.000154 & Deaminase \\
\hline L7N2D7 & Uncharacterized protein & 0.408 & 0.020565 & - \\
\hline P00688 & Pancreatic alpha-amylase & 0.4 & 0.000298 & Amylase \\
\hline B2RTM0 & Histone $\mathrm{H} 4$ & 0.383 & 0.01015 & Metalloprotease \\
\hline Q683Y7 & Immunoglobulin heavy chain variable region (Fragment) & 0.323 & 0.017091 & Immunoglobulin \\
\hline A0A075B677 & Immunoglobulin kappa variable $4-53$ & 0.322 & $2.75 \mathrm{E}-05$ & Immunoglobulin \\
\hline Q9Z0Y2 & Phospholipase A2 & 0.273 & 0.001599 & Phospholipase \\
\hline O88952 & Protein lin-7 homolog $\mathrm{C}$ & 0.268 & 0.004373 & Cell junction \\
\hline Q6P8U6 & Pancreatic triacylglycerol lipase & 0.255 & 0.000247 & Lipase \\
\hline
\end{tabular}

- Ratios $>2$ or $<0.5$ 
Figures

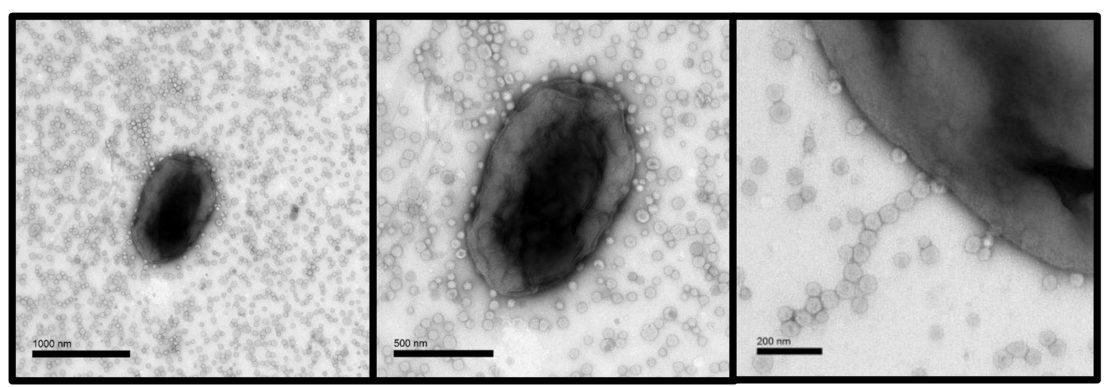

\section{Figure 1}

Release of BEVs from the cell surface of Bt into the external milieu. The cells were grown in BHI to early stationary phase and visualised by negative staining electron microscopy. 
a
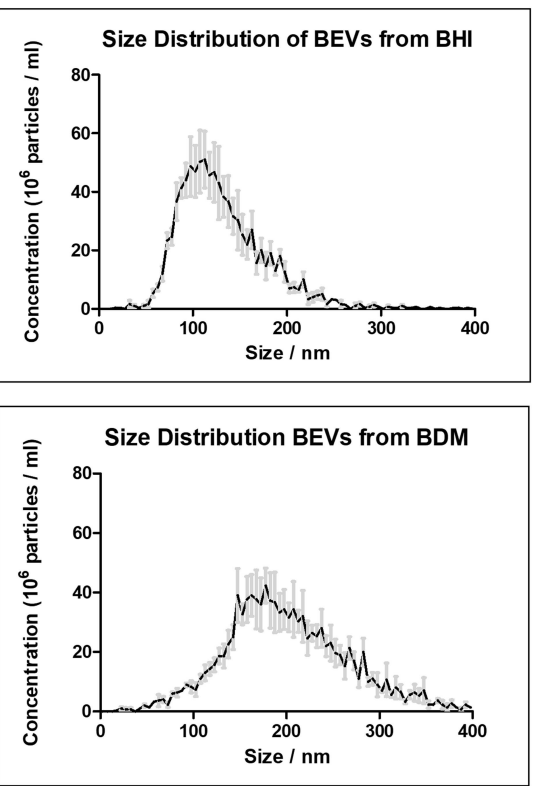

b
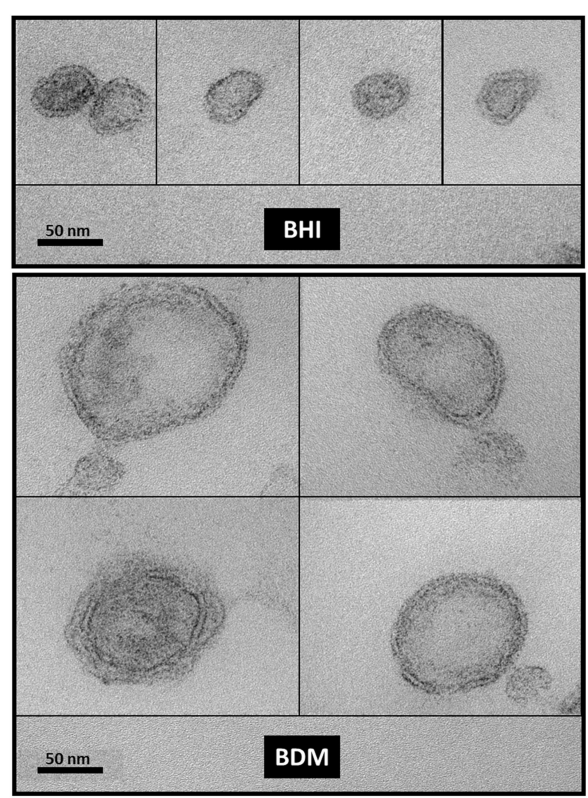

\section{Figure 2}

Impact of nutrient availability on BEV structure (a) Nanoparticle tracking analysis of BEVs suspensions obtained from Bt grown in nutrient-rich (BHI) or nutrientpoor (BDM) culture media $(n=3)$. (b) EM images of $\mathrm{BEV}$ s derived from Bt cells grown in complex medium $\mathrm{BHI}$ or defined medium BDM. 


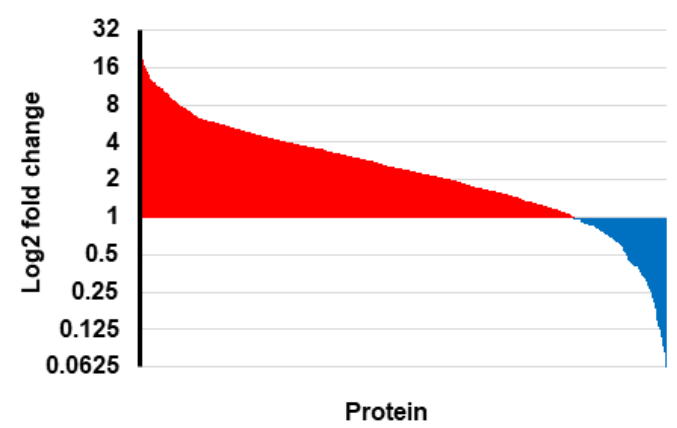

b

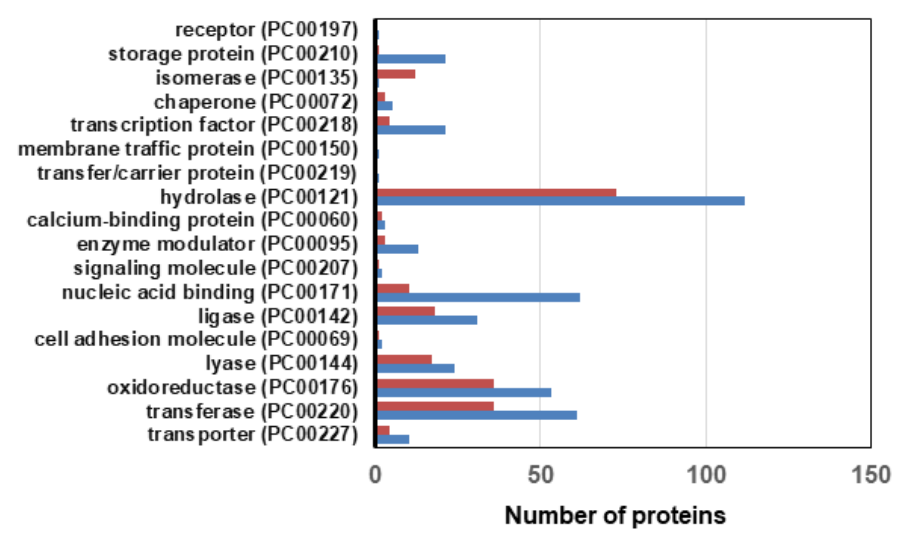

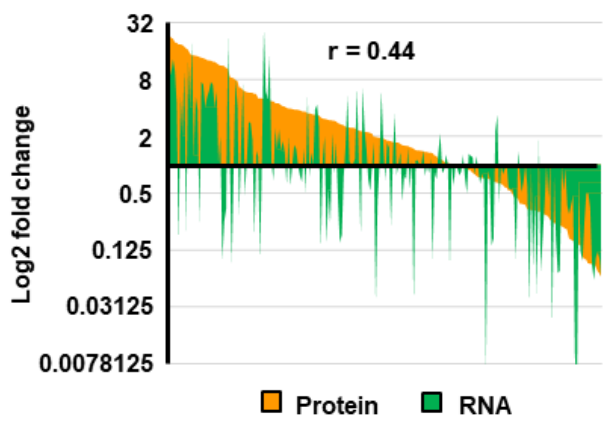

\section{Figure 3}

Proteomic profiles of BEVs produced in nutrient-rich and nutrient-poor media (a) Abundance ratio of each of the 1,438 proteins identified in protein profiling of BEVs obtained in BDM versus $\mathrm{BH}$. Proteins with a ratio higher than 1 are marked in red and those with a ratio below 1 are indicated in blue. (b) Proteins displaying an increased abundance (fold change $>3$ ) were categorized according to universal gene ontology (GO) annotations: Blue bars represent the total number of protein representing each category in BEVs: Orange bars represent the number of proteins of each category in BEVs that are $>3$ fold more abundant in BDM vs BHI. (c) Impact of growth medium on BEV proteome and parent cell transcriptome. Correlation between the abundance ratios of each of the 250 most abundant proteins (highest peptide spectrum match PSM) and the level of expression of the corresponding gene (Sonnenburg et al., 2018) identified in rich $(\mathrm{BHI})$ versus minimal defined (BDM) growth media. 
a

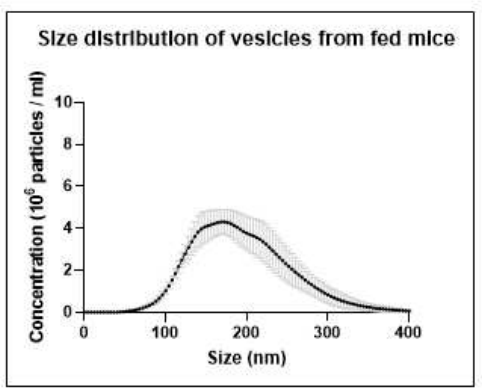

Size distribution of vesicles from fasted mice

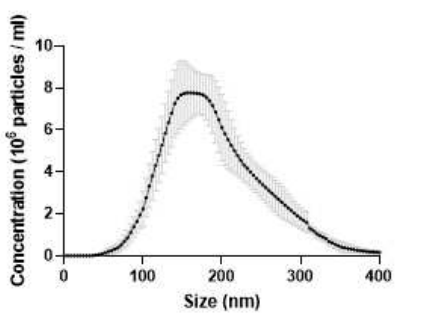

b
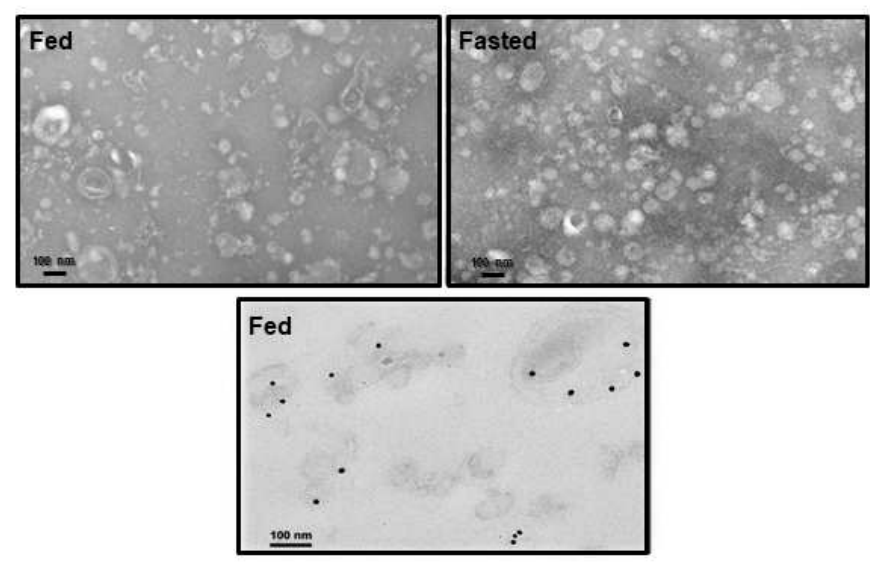

C

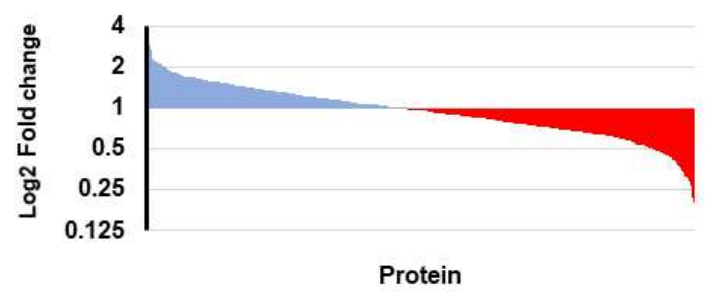

\section{Figure 4}

Structure and protein composition of BEVs produced in the mouse GIT. (a) Size distribution of extracellular vesicles produced in the caecum of germfree mice mono-colonised with Bt. The vesicles were extracted from caecal contents of mice either fed ad libitum or fasted for $16 \mathrm{~h}$ ( $\mathrm{n}=5 \mathrm{ea}$.). (b) TEM images of vesicles extracted from the caecum of fed or fasted mice. Lower panel shows immunodetection of Bt BEVs from fed mice using an in-house generated rabbit anti-Bt OmpA antiserum and colloidal gold anti-rabbit Ig. Scale bar $=100 \mathrm{~nm}$. (c) Comparison of the abundance of each of the 558 proteins identified in BEVs extracted from fasted versus fed mice. Proteins with a ratio higher than 1 are marked in red and those with a ratio below 1 are indicated in blue. 


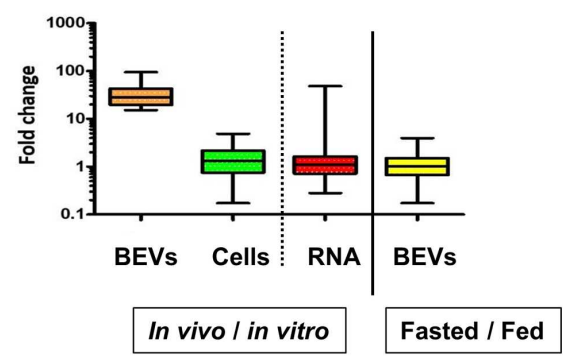

C

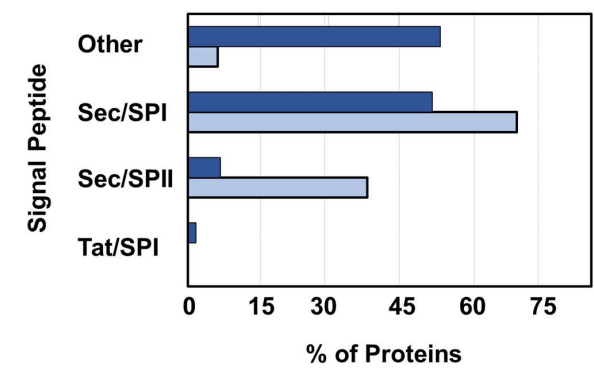

b

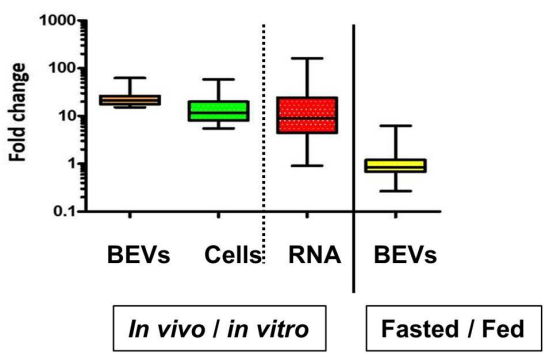

\section{Figure 5}

Proteins enriched in BEVs produced in the mouse GIT. The 102 proteins found to be enriched in BEVs in vivo (fold change $\geq 15$, Table S2), were divided into two groups based upon comparing their levels in BEVs versus parental cells. (a) 66 proteins had a less than 5 -fold increase in abundance in the parent cells in vivo (Table S2) are combined in (a), and results of the 36 proteins with a greater than 5 -fold change in the parent cells in vivo (Table S2) are combined in (b). For the two groups of proteins in (a) and (b), their expression is compared to that of the mRNA expression level of the corresponding gene in cells grown in similar conditions [29]. The impact of food withdrawal (Fasted/Fed) on the abundance of proteins in the two groups is also shown. (c) SignalP-5.0 Server at http://www.cbs.dtu.dk/services/SignalP/ was used to predict the presence of different types of signal peptides present amongst the two sets of enriched proteins. The 66 set is represented by dark blue bars and the 36 set of enriched proteins is represented by light blue bars. Sec/SPI are secretory signal peptides transported by the Sec translocon and cleaved by Signal Peptidase l; Sec/SPII are lipoprotein signal peptides transported by the Sec translocon and cleaved by Signal Peptidase II; Tat/SPI are Tat signal peptides transported by the Tat translocon and cleaved by Signal Peptidase I; "Other" are predicted secreted proteins translocated by uncharacterized secretion pathways. 


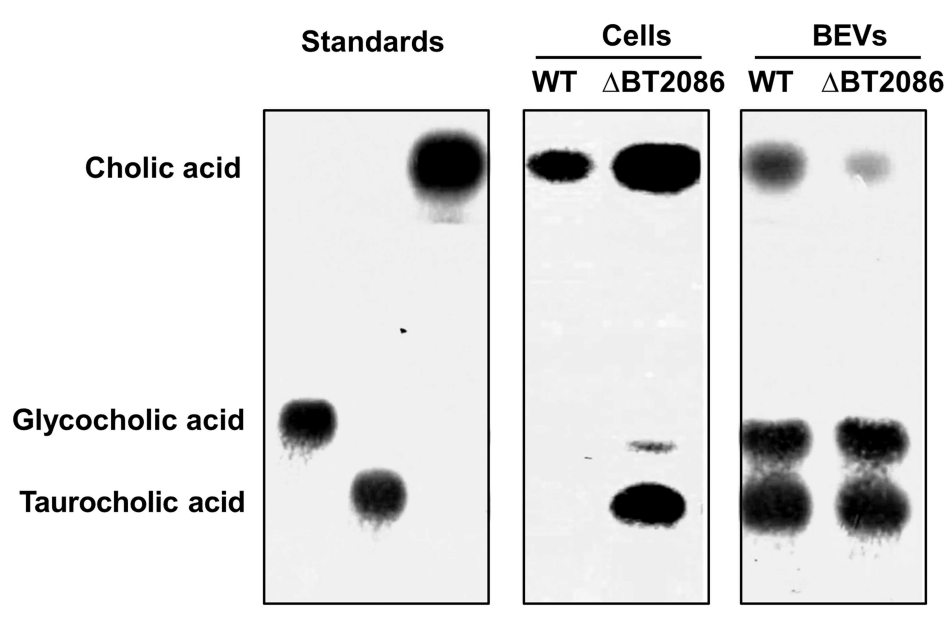

\section{Figure 6}

Bile salt hydrolase activity in Bt and BEVs. Thin layer chromatography was used to confirm BSH activity and substrate specificity of the BSH encoded by BT_2086 present in Bt cells and BEVs obtained after growth in BHI. Cholic acid [CA], taurocholic acid [TCA] and glycocholic acid [GCA] standards were incubated with whole cells or BEVs from wild type (WT) or a Bt BSH1 deletion mutant (ABT_2086) for 24h at $370 \mathrm{C}$ after which supernatants were spotted onto a silica gel plates. The plate was inserted into a TLC chamber, run for 40 min and stained with phosphomolybdic acid.

\section{Supplementary Files}

This is a list of supplementary files associated with this preprint. Click to download.

- TableS1.pdf

- TableS2RGS.xIsx 